Service social

\title{
Qualité de vie et drogues - Place aux jeunes, par l'Association des intervenants en toxicomanie du Québec, Chicoutimi, Gaëtan Morin, 1986, 262 pages.
}

\section{Jocelyn Lindsay}

Volume 35, numéro 3, 1986

Les jeunes et le travail social

URI : https://id.erudit.org/iderudit/706331ar

DOI : https://doi.org/10.7202/706331ar

Aller au sommaire du numéro

Éditeur(s)

École de service social de l'Université Laval

ISSN

1708-1734 (numérique)

Découvrir la revue

Citer ce compte rendu

Lindsay, J. (1986). Compte rendu de [Qualité de vie et drogues - Place aux jeunes, par l'Association des intervenants en toxicomanie du Québec, Chicoutimi, Gaëtan Morin, 1986, 262 pages.] Service social, 35(3), 487-488.

https://doi.org/10.7202/706331ar d'utilisation que vous pouvez consulter en ligne.

https://apropos.erudit.org/fr/usagers/politique-dutilisation/ 
de l'imagination ". Dans cette perspective, de dire l'auteure, " seuls comptent les actes et les conduites, jamais les significations " (p. 193).

Dans le secteur de la justice des mineurs, ce sera toujours la simulation qui primera : on ne croit pas les femmes et les enfants. Pourtant, conclut l'auteure : "Reconnaître que l'autre souffre, qu'il est profondément triste, plus encore qu'il aspire à mourir, c'est admettre l'authenticité de sa douleur et donc, lui donner une chance de parler, d'être entendu pour sa vraie peine, lui permettre de vivre. Refuser a priori cette dimension revient à lui ôter le droit d'une expression libre." (P. 203.)

Béatrice Koeppel a réussi à écrire un livre qui se lit comme un roman, tout en «brassant » de grosses théories qui la rapprochent des analyses de discours de Foucault et de l'anti-psychiatrie. Aux informations provenant du dossier de Marguerite, elle ajoute celles contenues dans les entrevues menées auprès de personnes qui l'ont connue. À ces diverses voix qui se font entendre, s'ajoute celle de Marguerite qui s'exprime dans les lettres qu'elle adressait à la Directrice de l'établissement et que l'auteure a reproduites sans aucun commentaire, fidèle à sa démarche de fournir une biographie institutionnelle et non personnelle. Ce sont des discours qui dominent la justice des mineures qu'elle rend compte et non de celui du sujet. À cela, s'ajoutent les quelques incursions personnelles de l'auteure dans son propre discours "scientifique".

Bien que rendant compte d'une réalité française, et que l'on soit ou non en accord avec le recours à la notion d'hystérie pour formuler une critique, ce livre ouvre des perspectives intéressantes et stimulantes pour questionner notre propre système d'aide à la jeunesse et le traitement fait à la déviance féminine puisque, dans ces domaines, les perspectives convergent sur de nombreux points.

Centre de services sociaux de Québec.

Lise BINET

Qualité de vie et drogues - Place aux jeunes, par l'Association des intervenants en toxicomanie du Québec, Chicoutimi, Gaëtan Morin, 1986, 262 pages.

Ce volume constitue les Actes du quatorzième colloque de l'Association des intervenants en toxicomanie du Québec, qui s'est tenu à Longueuil en mai 1985, pendant l'Année internationale de la jeunesse. Dans son organisation, l'ouvrage suit les différentes catégories de présentations annoncées dans le programme.

On trouve d'abord les textes des trois conférences principales. Jeanned'Arc Vaillant, alors sous-ministre adjointe, présente le projet d'orientations du Ministère des affaires sociales (Québec) à l'égard de l'usage et de l'abus des psychotropes. Roland Reumond aborde différentes stratégies d'éducation et de prévention développées en Belgique. Enfin, Marie-Andrée Bertrand questionne 
les intérêts professionnels et corporatistes comme obstacles premiers au changement des lois sur les drogues et à l'utilisation efficace des ressources pour toxicomanes.

Un second groupe de textes (Commission I) se rapporte aux réponses institutionnelles données aux jeunes aux prises avec des problèmes d'alcool et de drogue. Les uns touchent des ressources de réadaptation, les autres des actions judiciaires, et les derniers des interventions en milieu scolaire. C'est sûrement dans cette partie qu'un praticien trouvera le plus de matière pertinente.

La Commission II abordait la consommation de substances toxiques sous l'angle de la possibilité de choix et des facteurs qui peuvent influencer ceux-ci. On retrouve concrètement, dans ce troisième groupe de texte, outre une brève synthèse d'atelier sur l'influence des médias d'information sur les jeunes et les intervenants, deux présentations qui mettent en relation le comportement suicidaire et la toxicomanie.

La troisième Commission élargissait le débat par l'examen de quelques volets additionnels. Deux projets spécifiques et la possibilité d'un emploi sont présentés comme des alternatives à la consommation pouvant être proposées aux jeunes. Les autres ateliers ont touché l'implication communautaire bénévole, le rôle de la famille et l'entraide par les pairs.

Enfin, la Commission IV apportait un regard prospectif, et on retrouve des textes sur les valeurs des jeunes et sur leurs modèles d'identification.

Comme on peut s'y attendre, toutes les communications faites au colloque ne se retrouvent pas dans ce volume, et celles qui y sont varient en longueur et en qualité ; en ce sens, l'ouvrage est d'abord utile aux participants. Néanmoins, quelques textes sur l'intervention (dans les Commissions I et III) sont particulièrement intéressants. De plus, cet ouvrage a le mérite de faire mieux connaître l'Association des intervenants en toxicomanie du Québec.

École de service social,

Jocelyn LINDSAY

Université Laval.

Réussir sa jeunesse - Réflexions du Congrès international sur la jeunesse, textes choisis et présentés par Simone Piuze, Montréal, Stanké, 1986, 162 pages.

En novembre 1985, mille professionnels de toutes les régions du Canada participaient, à Montréal, à un congrès parrainé par les centres de jeunesse Shawbridge et l'Institut Philippe-Pinel, sous le thème "Jeunesse en devenir... se responsabiliser ". Madame Suzanne Piuze a choisi, parmi les cent trois conférences, celles qui lui semblaient les plus représentatives.

Le produit en est donc un volume contenant treize chapitres qui touchent autant de problématiques liées à la jeunesse. Les chapitres sont d'une longueur inégale (de 7 à 22 pages) et ont comme auteur la personne qui a fait la 